\section{A Miniature Instrumented Sphere to Understand Impacts Created by Mechanical Blueberry Harvesters}

\author{
Changying $\mathrm{Li}^{1,4}$, Pengcheng $\mathrm{Yu}^{1}$, Fumiomi Takeda ${ }^{2}$, \\ and Gerard Krewer ${ }^{3}$
}

AdDitional INDEx words. Vaccinium, Smart Berry, sensor, bruising, rotary, slapper

SUMmARY. The majority of U.S. northern highbush blueberry (Vaccinium corymbosum) and southern highbush blueberry (V. corymbosum hybrids) for the fresh market is hand harvested because of the high bruising damage to the fruit caused by current machine harvesters. To reduce bruising, it is important to understand how the harvester's machine parts interact with the fruit. A miniature instrumented sphere, hereafter referred to as Smart Berry, was developed to mimic a blueberry (Vaccinium species and hybrids) fruit and to quantitatively measure mechanical impacts experienced by a real blueberry fruit during mechanical harvesting. The Smart Berry sensor recorded impacts using three single-axis accelerometers with a maximum sampling frequency of $3 \mathrm{kHz}$ and $\pm 500 g_{n}$ sensing range. Calibration tests showed that the maximum error of the measurement was $0.53 \%$ of the output span. The diameter of the sensor ( 1 inch) was only half of that for the current smallest instrumented sphere on the market. Used together with a close-up video, the fully calibrated sensors were used to identify and measure mechanical impacts occurring in a commercial rotary blueberry harvester. The data suggested that the catch pan created the largest single mechanical impacts. Thus, reducing the drop height or padding the surface could be effective measures to reduce bruising damage caused by the catch pans. The Smart Berry was also used to compare harvesters with two different detaching mechanisms. The rotary detaching mechanism created significantly fewer and lower-magnitude impacts than the slapper mechanism $(P \leq$ 0.05). Manual drop tests demonstrated that the impact data recorded by the Smart Berry can be correlated with bruising damage experienced by blueberry fruit. Taken together, the data can be used to improve the design of the current machine harvesters for reduction of bruising damage to blueberry fruit destined for the fresh market, and potentially lead to enhanced highbush blueberry production efficiency in the long run.

$\mathrm{W}$ ith roughly 511 million pounds of blueberry accounting for $60 \%$ of world production in 2011, the United States is the largest blueberry-producing country in the world (U.S. Department of Agriculture, 2012). Although the blueberry for the processing market in the United States is mostly machine harvested, $70 \%$ of fresh-market blueberries are hand harvested. Hand harvest is labor intensive and costly (Brown, 1983), thus quickly becoming a major bottleneck for U.S. fresh-market blueberry production. There is a great demand to better adapt current machine harvesting technology for fresh-market blueberry, especially in areas where soft-textured southern highbush $(\mathrm{SH})$ blueberry is grown in hot and humid environments where fruit soften quickly upon reaching full maturity. Current mechanical harvesters originally intended for processed blueberries cause unacceptable levels of bruising for the fresh-market $\mathrm{SH}$ blueberry. Several studies have shown that bruising damage of blueberry fruit harvested by mechanical harvesters was substantially higher ( $55 \%$ to $78 \%$ ) than that of fruit harvested by hand (23\%) (Brown et al., 1996; Peterson et al., 1997). Clearly, the key to adapting the mechanical harvest to freshmarket blueberry is to reduce bruising to fruit by the machine. To achieve this goal, one critical step is to first understand how blueberry fruit interacts with the harvester; i.e., how many mechanical impacts are created, where they are created, and their magnitude.

To answer these questions, we designed a miniature instrumented sphere (Smart Berry) to mimic a real blueberry fruit by recording all significant impacts as it passes through the harvesting process. The Smart Berry is essentially a data logger with accelerometers, internal power supply, memory, and microprocessor integrated into a l-inch-diameter sphere cast in silicone rubber. Although various types of instrumented spheres have been developed and used for specific purposes in the past two decades, most have been used in impact evaluation of large fruit and vegetables, such as apples (Malus $\times$ domestica), onions (Allium cepa), and potatoes (Solanum tuberosum), in the packing house, none has been used for small fruit because of their comparatively large size and weight. In contrast, the Smart Berry is only half the size and a fraction of the weight of the current smallest instrumented sphere available on the market, such as Impact Recording Device (Techmark, Lansing, MI)

This paper summarizes the hardware and software of the instrumented sphere sensing system; its application in evaluating a rotary and a slapper harvester design; and how the impact data collected could be translated into fruit bruising rates for two $\mathrm{SH}$ blueberries ('Scintilla' and FL 05-528). Technical details of sensor design, development, and evaluation have been published in specialized papers ( $\mathrm{Yu}$ et al., 2011a, 2011b, 2012).

\section{Smart Berry}

Overall, the Smart Berry sensing system consists of three essential components: the sensor, the interface box, and the software program on a personal computer for data retrieval and analysis. The Smart Berry itself has four main components (i.e., accelerometer, microprocessor, memory, and battery), making it an independent impact data collection and recording device. Impact data are measured by three accelerometers (sensing range $\pm 500 g_{\mathrm{n}}$ ) in acceleration values $\left(1 g_{n}=9.81 \mathrm{~m} \cdot \mathrm{s}^{-2}\right)$ from three orthogonal directions. The microprocessor manages the process of

\begin{tabular}{llll}
\hline $\begin{array}{l}\text { Units } \\
\begin{array}{l}\text { To convert U.S. to SI, } \\
\text { multiply by }\end{array}\end{array}$ & U.S. unit & SI unit & $\begin{array}{l}\text { To convert SI to U.S., } \\
\text { multiply by }\end{array}$ \\
\hline 0.3048 & $\mathrm{ft}$ & $\mathrm{m}$ & 3.2808 \\
2.54 & inch(es) & $\mathrm{cm}$ & 0.3937 \\
0.4536 & $\mathrm{lb}$ & $\mathrm{kg}$ & 2.2046 \\
1.6093 & $\mathrm{mph}$ & $\mathrm{km} \cdot \mathrm{h}^{-1}$ & 0.6214
\end{tabular}


data collection and recording, including analog-to-digital conversion, computation, fast data saving and retrieving, creation of a real-time clock, and communication with the interface box and computer. The maximum sampling frequency is $3 \mathrm{kHz}$. All impact data are saved in a memory chip with a capacity of $128 \mathrm{kB}$, equivalent to more than 9000 impact data sets. A lithium-ion rechargeable battery ( $75 \mathrm{~mA}$-h capacity) is used to power the entire circuit at $3.3 \mathrm{~V}$. A circuit board, which holds the four major components and other peripheral electronics, was cast in silicon rubber to form a 1 -inch-diameter sphere (Fig. 1).

An interface box serves as an intermediate device to connect the sensor with the computer for data downloading and sensor configuration. Another important function of the interface box is to recharge the lithium-ion battery in the Smart Berry while the sensor is connected with the interface box.

Each of the three components of the sensing system has its own software program: the sensor program, the interface box program, and the computer graphical user interface program (Yu et al., 201lb). The software programs for the sensor and the interface box act like operating systems for each of the hardware systems, while the computer software provides a graphical user interface for sensor

\footnotetext{
This paper was part of the workshop "Mechanized Harvest of Blueberries for the Fresh Market" held 2 Aug. 2012 at the ASHS Conference, Miami, FL, and sponsored by the Viticulture and Small Fruits (VSF) Working Group.

This project was funded by the U.S. Department of Agriculture National Institute of Food and Agriculture Specialty Crop Research Initiative (Award No. 2008-51180-19579).

We thank Gary Burnham and Tim Rutland's technical support. We also appreciate the assistance provided by John Ed Smith and Robert D. Stanaland during the field tests. The Triple R. Farm, the Allen Blueberry Farm, and the DHL farm provided mechanical harvesters and blueberry plants for the field test, to which we are grateful. We also thank Oxbo International Inc. for leasing a rotary harvester for preliminary tests on the early stage of this study. We are grateful for the comments and suggestions made by Harald Scherm, Renfu Lu, and Anish Malladi to improve the manuscript.

${ }^{1}$ College of Engineering, 712F Boyd Graduate Studies, University of Georgia, Athens, GA 30602

${ }^{2}$ Appalachian Fruit Research Station, USDA-ARS, 2217 Wiltshire Road, Kearneysville, WV 25430

${ }^{3}$ Department of Horticulture, University of Georgia, Tifton, Tifton Campus, 2360 Rainwater Road, GA 31793. Current address: Krewer Consulting, LLC, 550 Mary's Drive, Woodbine, GA 31569.

${ }^{4}$ Corresponding author. E-mail: cyli@uga.edu.
}

configuration, data download and processing, and visual display of the data (Fig. 2). The PICBasic Pro (PBP) (microEngineering Labs, Colorado Springs, CO) programming language was used to develop programs at the microcontroller level for both the sensor and the interface box. The graphic user interface program on the computer was designed using the graphical programming language LabVIEW (V8.2; National Instruments, Austin, TX).

The Smart Berry sensor was calibrated using a centrifuge (model 5810; Eppendorf, Hamburg, Germany), which provided stable acceleration at the centripetal direction at a given rotational speed (Yu et al., 201la). The acceleration values created by the centrifuge were compared with the data recorded by the sensor mounted inside the centrifuge. A linear relationship between the centrifuge and sensor was observed with coefficient of determination $\left(r^{2}\right)$ of 1 and standard error of prediction of $0.60 g_{n}$. The maximum error of the

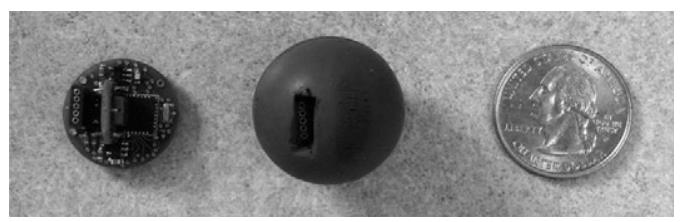

Fig. 1. The circuit board (left) and the Smart Berry sensor, a miniature instrumented sphere (middle), compared with a quarter coin (right).

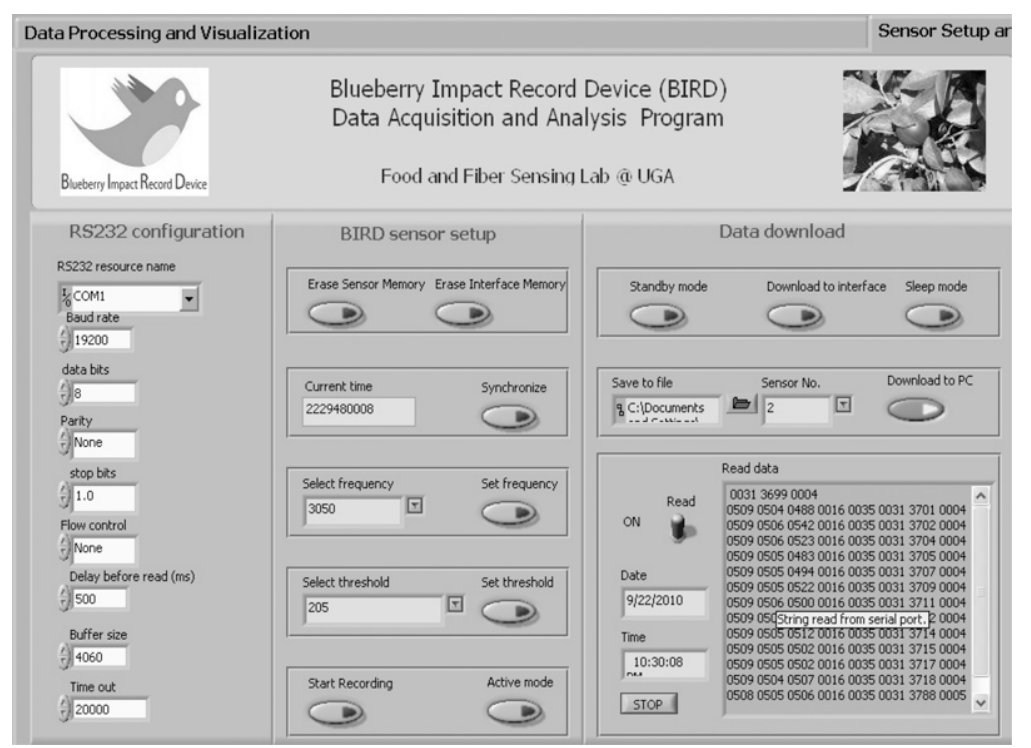

Fig. 2. The graphical user interface (GUI) of the computer program for sensor configuration, data download, and processing (Yu et al., 2011b). 
to $420 \mathrm{rpm}$. These settings were recommended by the manufacturer of the harvester and are adopted by most commercial blueberry farms.

The Smart Berry was attached to blueberry bushes at $1.5 \mathrm{~m}$ above the ground using a specially designed three-legged cradle (Fig. 3). The bushes were mature plants with multiple canes and they were similar in height $(1.8-2.5 \mathrm{~m})$, structure, and limb density. The harvesting process was captured with a video recorder (T2i; Canon, Tokyo, Japan) to relate the location of the sensor with the impact data and to identify critical control points that created most impacts and potential bruising. A stopwatch and the clocks in the sensor and the video recorder were synchronized with the central processing unit clock on a laptop before each test. On the basis of the time stamp of each impact, impacts recorded by the sensor were related and identified in the video footage. A total of eight harvesting sessions were recorded successfully, which clearly documented the track of the sensor during the harvesting process. Data collected by the sensor were processed using a customized computer program.

The impact data collected by the sensor helped to answer several important questions that were elusive to growers and researchers in the past. For instance, how long is a typical harvesting process for an individual blueberry fruit? How many impacts can be created during the harvesting process? How large are these impacts? And where are these impacts created during the process of interacting with the machine harvester?

From the data recorded by the Smart Berry during one particular harvesting event, the elapsed time was about $8 \mathrm{~s}$ from the time when the sensor was agitated by the mechanical force of the blueberry detachment mechanism to the time when it dropped into a lug box. During this period, 14 impacts were recorded by the sensor. The largest impact occurred at $0.31 \mathrm{~s}$ with a magnitude of $267 g_{\mathrm{n}}$. The synchronized video footage showed that this impact occurred when the sensor dropped on the catch pan. Only a minor impact $\left(27 g_{n}\right)$ was recorded from time 0 to time $0.31 \mathrm{~s}$, which suggested that the impact created by interacting with beaters or bush limbs was not as large as previously thought. Observed under a finer resolution, each impact lasted for only 7 to $8 \mathrm{~ms}$. Most impacts occurred when the detached sensor contacted machine surfaces within the harvester tunnel. Significant impacts were also observed when the sensor dropped onto an empty lug from the conveyor belt (Yu et al., 2012).

On the basis of the close-up video, five types of contacting surfaces (shaking rods, the tunnel, the catch pan, the conveyer belt, and the lug) were clearly identified in most or all of the video footage (Fig. 4). Our analyses revealed that the largest cumulative impacts were created by the catch pan, accounting for $31 \%$ of cumulative impacts from all contacting surfaces. The second largest impacts were created by the lug, contributing $24 \%$ of cumulative impacts. However, it should be noted that the impacts caused by the lug could be overestimated because the sensor dropped to the bottom of the lug in our field tests although in reality the majority of the fruit in a lug does not come in contact with the bottom of the lug but rather drop on top of other fruit. The conveyor belt and shaking rods were largely similar in creating impacts and contributed to about $13 \%$ of cumulative impacts. Cumulative impacts created by the tunnel constituted less than $7 \%$ of all impacts. The results suggest that blueberry fruit are more likely to collide directly with the catch pan and the conveyor belt than with

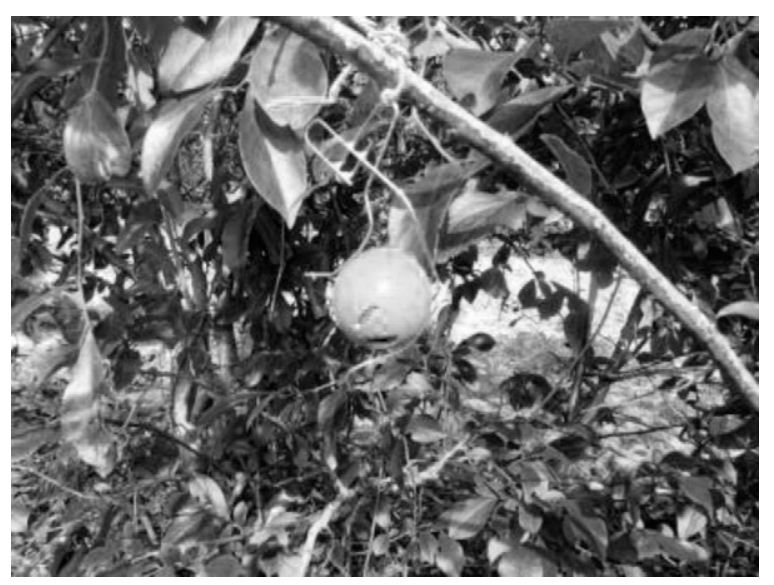

Fig. 3. An illustration of how the Smart Berry sensor, a miniature instrumented sphere, was attached to the southern highbush blueberry plant during the field tests.

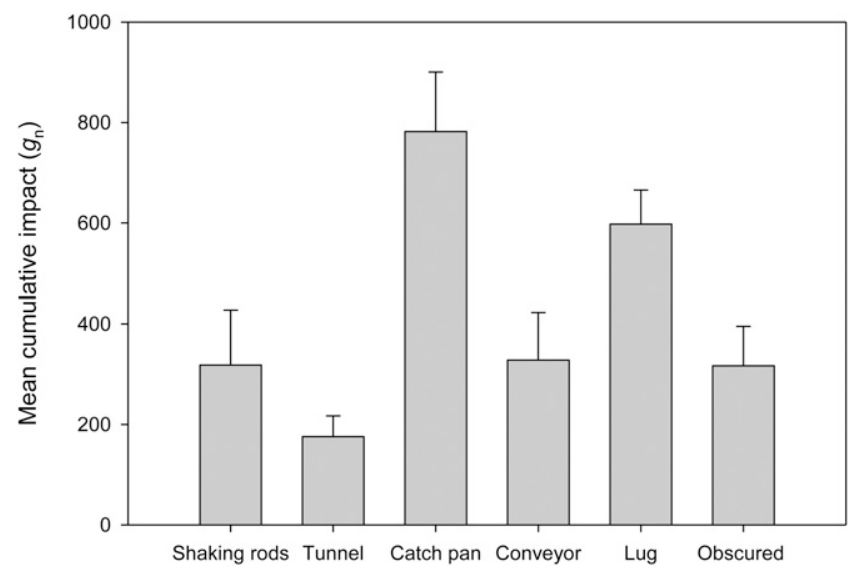

Fig. 4. Magnitude of cumulative impacts created by different mechanical components of the rotary harvester $(n=8)$. Data collected with the Smart Berry sensor, a miniature instrumented sphere, shown in Fig. 1. The "Lug" refers to the fruit collection box; the "Obscured" group refers to the impacts that were not clearly identified in the video footages; the "tunnel" refers to the harvester tunnel wall made of metal sheet. Error bars represent $S E ; 1 g_{n}=9.81 \mathrm{~m} \cdot \mathrm{s}^{-2}=32.185 \mathrm{ft} / \mathrm{s}^{2}$. 
the shaking rods and the side panels of the harvester tunnel. To reduce fruit bruising, padding the catch pans, lugs, and conveyer belt using soft cushioning materials could be one effective method (Brown et al., 1996; Fridley et al., 1964). Another effective approach is to add a deflecting mechanism or to reduce the drop height of fruit from the conveyer belt to the lug, as done in apple harvesting (Peterson and Wolford, 2003) and in the V45 blueberry harvester (Peterson et al., 1997). A caveat should be added that because of the discrepancy in size and weight between the Smart Berry and the real blueberry fruit, the trajectory measured by the sensor may not exactly represent the true trajectory of a real blueberry fruit. An effort to improve the current sensor to better resemble a real blueberry fruit is underway. In addition, the current study did not consider the effect of plant shape, denseness, and height of different $\mathrm{SH}$ blueberry on the impact values, which should be examined in future studies.

\section{Comparison of rotary and slapper harvesters}

In the second evaluation, the Smart Berry was used to compare commonly used rotary harvester (Oxbo) and slapper harvester (BEI, South Haven, MI) designs in terms of the impacts they created. The study was conducted in the Fall 2010. Blueberry bushes that had been harvested in the spring and were similar in height (1.8-2.5 m), limb density, and ground conditions were selected for the study. Each row had roughly 40 to 50 bushes (about $50 \mathrm{~m}$ ), among which 8 to 10 bushes were selected to attach the sensors with 5 bushes separating those that were mounted with the Smart Berry sensors. During the field tests, the sensors were attached on blueberry bushes at a height of $150 \mathrm{~cm}$ above the ground. The harvesters were operated in a manner that was typical for commercial operations with beater speeds of 420 and $166 \mathrm{rpm}$ for rotary and slapper harvesters, respectively. The ground speed of both harvesters was set at $\mathrm{l} \mathrm{mph}$. The data were considered valid only when the sensor fell into the lug after going through the harvester.

A consistent pattern was observed from the data recorded by the
Smart Berry, whereby both the number and magnitude of impacts created by the rotary harvester were significantly lower than those created by the slapper harvester $(P \leq 0.05)$ (Table 1$)$. On average, for each harvesting event, the slapper harvester generated 10 more impacts and $100 g_{n}$ larger maximum impacts than the rotary harvester.

These differences were caused primarily by the different agitating mechanisms and surface properties of the harvesters. The rotary harvester has two spindles of beaters that vibrate to shake off ripe blueberry fruit from the bushes. The slapper harvester, in contrast, uses two columns of beater bars to swing to opposite directions like "slapping" on blueberry bushes from both sides to remove the blueberry fruit from the bushes. The slapper harvester had longer impact-generating periods during the overall harvesting process than the rotary harvester, which explains why it created a larger number of impacts. The shaking rods in the rotary harvester vibrated, but did not actively beat bushes. The beaters in the slapper actively interacted with the bush. In addition, the conveyer belt of the rotary harvester was made of plastic (polypropylene) and was less damaging than that of the slapper, which was made of steel. Finally, the drop height from the conveyer belt to the lug in the rotary harvester (about $35 \mathrm{~cm}$ ) was less than that in the slapper (about $80 \mathrm{~cm}$ ). All these differences led to the larger impacts (both in number and magnitude) with the slapper.

\section{Relating impact data with fruit bruising}

The acceleration values measured by the Smart Berry delineated the relative differences of harshness from different types of harvesting processes and contacting surfaces.
The magnitude of the impacts, however, did not directly represent the impact that a blueberry fruit experienced. Therefore, it would be more meaningful if impact values described by acceleration values were correlated with bruising rate of blueberry fruit. To address this need, both blueberry fruit and the sensor were dropped from the same heights onto the same contacting surfaces. Bruising damage and impact data recorded by the sensor were compared and related.

Two SH blueberries (soft-textured cultivar Scintilla and firm-textured selection FL 05-528) were selected for this study. Hand-harvested blueberry fruit (three replicates of 50 fruit) was dropped individually onto a hard plastic surface (Plexiglas ${ }^{\circledR}$; Arkema, Exton, PA) from two heights $(60$ and $120 \mathrm{~cm})$ on the day of harvesting. An additional 50 fruit of 'Scintilla' and FL 05-528 were not dropped and served as control samples. After $24 \mathrm{~h}$, both the dropped and undropped (control) fruit were sliced through the equator and evaluated for surface area of discolored zones. Fruit having a discolored area larger than $25 \%$ of the sliced surface was rated as bruised. The Smart Berry was dropped onto the same hard plastic surfaces from the two heights, with 20 replicates for each height.

The drop tests data showed that the sensor recorded $604 g_{\mathrm{n}}$ when it was dropped on the plastic surface at $60 \mathrm{~cm}$, which corresponded to $44 \%$ bruising incidence for 'Scintilla' and $19 \%$ for FL $05-528$. At the $120-\mathrm{cm}$ drop height, the sensor recorded $814 g_{n}$, which paralleled with $76 \%$ and $31 \%$ bruising incidences for 'Scintilla' and FL 05-528, respectively. Clearly, both the impact values recorded by the Smart Berry and bruising incidences for 'Scintilla' and FL 05-528 fruit had a positive relationship with the drop height when both the sensor and the fruit were dropped onto a hard plastic surface. Therefore, the

Table 1. Comparison of rotary and slapper harvesters in number of impacts and magnitude of the maximum impacts $(n=20)$. Data were collected with the Smart Berry sensor, a miniature instrumented sphere, shown in Fig. 1.

\begin{tabular}{lcc}
\hline & Impacts $[$ mean \pm SE $($ no. $)]$ & Maximum impact $\left[\text { mean } \pm \text { SE }\left(g_{\mathrm{n}}\right)\right]^{\mathrm{z}}$ \\
\hline Rotary & $22.7 \pm 1.4 \mathrm{~b}^{\mathrm{y}}$ & $352.2 \pm 20.8 \mathrm{~b}$ \\
Slapper & $31.8 \pm 1.3 \mathrm{a}$ & $458.5 \pm 33.2 \mathrm{a}$ \\
\hline
\end{tabular}

${ }^{\mathrm{z}} 1 g_{\mathrm{n}}=9.81 \mathrm{~m} \cdot \mathrm{s}^{-2}=32.185 \mathrm{ft} / \mathrm{s}^{2}$.

${ }^{y}$ Mean separation by least significant difference test. Means followed by a different letter are significantly different at $P \leq 0.05$. 
impact values recorded by the sensor can be related to the bruising incidences of a particular SH blueberry using this method on the hard plastic surface. However, cautions should be made that this relationship does not take contacting surface properties into consideration. Other methods such as using both the velocity change and impact graph could be used to establish bruising probability lines. In addition, only two drop heights were used for the current study and more than two drop heights should be used to make the relation more robust in future studies.

\section{Conclusions}

The Smart Berry enables quantitative measurements of the interactions between the sensor, the blueberry plant, and machine harvesters, which had not been possible previously. Coupled with close-up video footage, the data from the sensor revealed that the catch pans of the rotary harvester created not only the largest single mechanical impact, but also the largest cumulative mechanical impacts. The findings suggest that reducing the drop height or padding the surface of the catch pans could be effective measures to reduce potential bruising damage. The data from the sensor also showed that the rotary detaching mechanism created fewer and lower-magnitude impacts than the slapper mechanism, suggesting current rotary harvester designs may be the better choice for machine harvesting $\mathrm{SH}$ blueberry destined for fresh market.

Relating the impact data of the sensor to the fruit bruising incidence values proved the usefulness of the sensor in evaluating bruise susceptibility of various SH blueberry. The current sensor is still larger and heavier than a typical blueberry fruit. An effort is underway in the development of a second-generation Smart Berry which is smaller and lighter and consequently may further improve performance. With the aid of the sensor, blueberry growers will be able to improve their harvesting procedures and the manufacturers of blueberry harvesters will be able to improve their current designs, which could lead to improved fruit quality and enhanced highbush blueberry production efficiency.

\section{Literature cited}

Brown, G.K. 1983. Status of harvest mechanization of horticultural crops, p. 78. In: ASAE Spec. Publ. 0383. Amer. Soc. Agr. Eng., St. Joseph, MI.

Brown, G.K., N.L. Schulte, E.J. Timm, R.M. Beaudry, D.L. Peterson, J.F. Hancock, and F. Takeda. 1996. Estimates of mechanization effects on fresh blueberry quality. Appl. Eng. Agr. 12:2126.
Fridley, R.B., H. Goehlich, L.L. Claypool, and P.A. Adrian. 1964. Factors affecting impact injury to mechanically harvested fruit. Trans. Amer. Soc. Agr. Biol. Eng. 7:409-411.

Peterson, D.L. and S.D. Wolford. 2003. Bin filler for fruit crops. U.S. Patent No. US6644905 Bl. U.S. Patent and Trademark Office, Washington, DC.

Peterson, D.L., S.D. Wolford, E.J. Timm, and F. Takeda. 1997. Fresh market quality blueberry harvester. Trans. Amer. Soc. Agr. Eng. 40:535-540.

U.S. Department of Agriculture. 2012. U.S. blueberry production and utilization (cultivated and wild), selected States, 1980-2011. 24 Jan. 2013. <http:// usda.mannlib.cornell.edu/MannUsda/ viewDocumentInfo.do? documentID= 1765>.

Yu, P., C. Li, G. Rains, and T. Hamrita. 201 la. Development of the Berry Impact Recording Device sensing system: Hardware design and calibration. Comput. Electron. Agr. 79:103-111.

Yu, P., C. Li, G. Rains, and T. Hamrita. 2011 b. Development of the Berry Impact Recording Device sensing system: Software. Comput. Electron. Agr. 77:195203.

Yu, P., C. Li, F. Takeda, G. Krewer, G. Rains, and T. Hamrita. 2012. Quantitative evaluation of a rotary blueberry mechanical harvester using a miniature instrumented sphere. Comput. Electron. Agr. 88:25-31. 